\title{
Supporting information for: Energy budget of liquid drop impact at maximum spreading: numerical simulations and experiments
}

Jae Bong Lee, ${ }^{\dagger}$ Dominique Derome, ${ }^{\ddagger}$ Ali Dolatabadi, and Jan Carmeliet ${ }^{*, \dagger, \ddagger}$

${ }^{\dagger}$ Chair of Building Physics, ETH Zurich, Stefano-Franscini-Platz 5, CH-8093 Zürich, Switzerland. ${ }^{*}$ Laboratory for Multiscale Studies for Building Physics, Swiss Federal Laboratories for Materials Science and Technology, EMPA, Überlandstrasse 129, CH-8600 Dbendorf, Switzerland. IDepartment of Mechanical and Industrial Engineering, Concordia University, Montreal, Quebec H3G1M8, Canada.

E-mail: carmeliet@arch.ethz.ch

Phone: +41 (0)4463328 55. Fax: +41 (0)446331041

Rescaling for maximum spreading diameter

We also plot the non-dimensional maximum spreading, with $D_{\max } /\left(D \cdot \operatorname{Re}^{1 / 5}\right)$ as function of $P=\mathrm{We} / \mathrm{Re}^{4 / 5}$ as suggested by Clanet et al. ${ }^{1}$ Figure $\mathrm{S} 1$ shows that this scaling, although predicting the behavior for water, does not predict the maximum spreading diameter for ethanol and glycerol droplets.

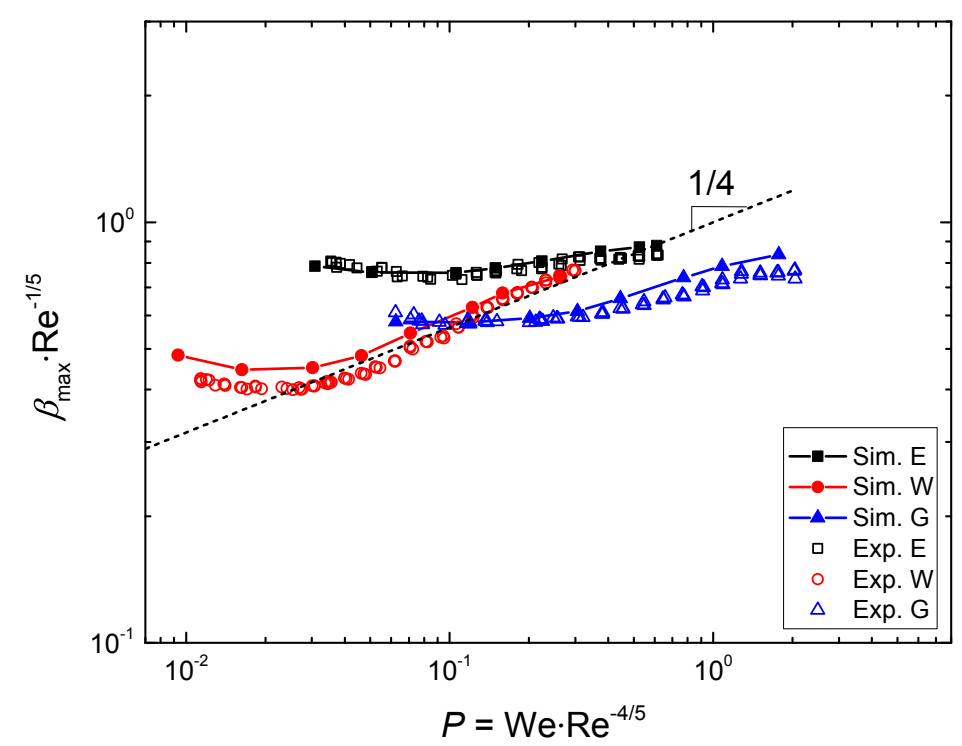

Fig. S1 Rescaled maximum spreading ratio as a function of the impact number $(P=$ We $\cdot \operatorname{Re}^{-4 / 5}$ ) as suggested by Clanet et al. ${ }^{1}$ 


\section{Reference}

(1) Clanet, C.; Béguin, C.; Richard, D.; Quéré, D. Maximal deformation of an impacting drop. Journal of Fluid Mechanics 2004, 517, 199-208. 